\title{
Prognostic Factors of Patients with Postinfantile Epilepsy and Multiple Independent Spike Foci on Electroencephalography
}

\author{
Juhyun Kong, MD¹, Yun-Jin Lee, MD¹, Ara Ko, MD¹, Young Mi Kim, MD², Gyu Min Yeon, MD³, \\ Sang Ook Nam, MD \\ 'Department of Pediatrics, Pusan National University Children's Hospital, Pusan National University School of Medicine, Yangsan, Korea \\ ${ }^{2}$ Department of Pediatrics, Pusan National University Hospital, Busan, Korea \\ ${ }^{3}$ Department of Pediatrics, Kosin University Gospel Hospital, Kosin University College of Medicine, Busan, Korea
}

Received: May 18, 2020

Revised: May 27, 2020

Accepted: May 31, 2020

Corresponding author:

Sang Ook Nam, MD

Department of Pediatrics, Pusan

National University Children's

Hospital, Pusan National University

School of Medicine, 20 Geumo-ro,

Mulgeum-eup, Yangsan 50612,

Korea

Tel: +82-55-360-2181

Fax: +82-55-360-2182

E-mail: neuroped@naver.com
Purpose: Multiple independent spike foci (MISF) have been reported to be associated with hypsarrhythmia and slow spikes and waves. However, some patients with MISF demonstrate a good prognosis, such as benign focal epilepsy. This study aimed to elucidate the prognosis of epileptic children with MISF and to analyze the prognostic factors.

Methods: The subjects were 115 epileptic children aged 1 to 18 years who visited Pusan National University Children's Hospital between November 2008 and July 2016 and in whom MISF were noted on electroencephalography. We excluded patients with infantile spasms, congenital metabolic diseases, neurodegenerative diseases, or post-encephalitic epilepsy. We retrospectively reviewed participants' clinical information. Seizure control was defined as no seizures over 6 months at the last visit. Prognostic factors were analyzed in the seizure control (group $A ; 84$ [73\%]) and no seizure control (group B; 31 [27\%]) groups.

Results: Generalized seizure ( $P=0.033)$, intellectual disability $(P<0.001)$, cerebral palsy $(P=0.046)$, and abnormal background activity and electrodecrements on electroencephalography $(P<0.001)$ were significantly more common in group B. No clinically significant abnormalities were noted on magnetic resonance imaging of the brain. The MISF improved on follow-up electroencephalography in $58(71.6 \%)$ patients in group A versus $10(35.7 \%)$ in group $B(P=0.002)$.

Conclusion: Despite MISF on electroencephalography, two-thirds of patients had a benign clinical course, particularly those with post-infantile epilepsy and no infantile spasms. The prognostic factors of poor outcomes were generalized seizures, intellectual disability, and abnormal background activity on electroencephalography.

Keywords: Child; Electroencephalography; Epilepsy; Prognosis

Copyright (C) 2020 Korean Child Neurology Society

This is an Open Access article distributed under the terms of the Creative Commons Attribution Non-Commercial License (http://creativecommons.org/licenses/by-nc/4.0/) which permits unrestricted non-commercial use, distribution, and reproduction in any medium, provided the original work is properly cited. 


\section{Introduction}

Historically, in the 1970s, the presence of multiple independent spike foci (MISF) on electroencephalography (EEG) was considered findings associated with hypsarrhythmia and slow spike and wave complexes, which were remarkable findings of infantile spasms and Lennox-Gaustaut syndrome (LGS) [1-3]. Blume [4] also described it was highly associated with intellectual abnormality despite a lack of infantile spasms or LGS. Thereafter, numerous studies on epileptic syndrome, infantile spasms, and LGS have led to their being widely accepted as epileptic syndromes distinctive from others; however, a few recent studies have aimed to reassess the significance of MISF in epilepsy.

Several recent studies proposed that patients for whom MISF are seen on EEG along with some characteristic clinical findings, who had poor prognosis and poor clinical course might be reclassified as having so-called severe epilepsy with MISF (SE-MISF) $[5,6]$. Nevertheless, it were not widely accepted yet and in the clinical setting, cases with unfavorable prognosis as well as favorable outcome despite MISF have been documented similar to those of patients with benign focal epilepsy. Here we aimed to analyze the prognosis of patients with post-infantile epilepsy with MISF and the prognostic factors associated with seizure control.

\section{Materials and Methods}

\section{Patients and method}

This retrospective study included patients aged 1 to 18 years who underwent an EEG study at the pediatric neurology clinic at Pusan National Children's Hospital between November 1, 2008 and July 31,2016 . All subjects were patients with epilepsy diagnosed by the presence of two or more episodes of unprovoked seizures and MISF on EEG at least once before and during treatment. We included patients whose follow-up duration after MISF was over 1 year. We excluded patients who were diagnosed with infantile spasms and an expected poor prognosis; those who had a seizure onset before 1 year; or those with inborn errors of metabolism, neurodegenerative disease, acute encephalitis, or bacterial meningitis.

\section{Method}

This retrospective cohort study investigated the clinical information and factors associated with prognosis through medical chart reviews. MISF on EEG is defined as asynchronous multifocal epileptiform discharge from more than three foci with bilateral distribution and no adjacent electrical field. The treatment result was assessed as seizure control or non-control. The definition of seizure control was freedom from seizures for prolonged more than 6 months at the last visit by June 31, 2017. The patients with seizure control were included in the seizure control group (group A), while those without seizure control were in the no seizure control group (group B). The groups were compared to identify the prognostic factors influencing the therapeutic result.

Clinical information included sex, age at seizure onset, age at EEG study showing MISF, birth history such as gestational age and perinatal problems, and family history of epilepsy or febrile convulsions. The seizure profiles that were related to a history of seizures included seizure type, status epilepticus, duration between first seizure and start of anti-epileptic drug (AED) therapy, seizure frequency during the most recent 3 months from the identification of MISF on EEG. Comorbid conditions included intellectual disability (developmental delay in early childhood, including a speech-language delay), autism spectrum disorder, cerebral palsy, genetic disorder, and lesions suspiciously associated with epilepsy on magnetic resonance imaging (MRI). Other abnormal EEG findings were also analyzed, including abnormal background activities, focal slow waves, and generalized epileptiform discharges. We reviewed follow-up EEG study, which were most recent study within 1 year from last visit and compared with clinical outcome, seizure control.

Data from statistical analysis are expressed as medians and interquartile ranges (IQRs) for continuous and ordinal variables, and as counts and percentages for categorical variables. The two groups were compared using the chi-square test or Fisher's exact tests for categorical and ordinal data, or the Mann-Whitney $U$ test for non-parametric continuous data. A P $<0.05$ was considered significant. R Statistics and Microsoft Excel was used for statistical analysis.

\section{Ethical statement}

This study protocol was approved by the Institutional Review Board of Yangsan Pusan National University Hospital (no. 052019-047). The need for informed consent was waived by the board.

\section{Results}

\section{Demographics}

A total of 394 of patients were identified with MISF on EEG between November 1, 2008 and July 31, 2016. Among them, 115 who were diagnosed with epilepsy, treated, and followed up for more than 1 year, and did not meet the exclusion criteria were included in the current study. The patients' demographic profiles are shown in Table 1. The study population included 68 male patients and 47 female patients (ratio, 1.45:1). The mean onset age was 
$4.88 \pm 2.99$ years, while the median age was 4.5 years (IQR, 2.29 to 6.88). The mean age at MISF on EEG was $8.08 \pm 3.53$ years, while the median age at MISF on EEG was 6 years (IQR, 5 to 8.67). Seizure type was generalized in 49 (43\%) patients and focal in 66 (57\%). Of them, 29 patients (25\%) had symptomatic epilepsy with lesions on brain MRI. Eight patients (7\%) had a history of perinatal problems, 68 (59\%) had intellectual disability, 34 (30\%) had cerebral palsy, and seven (6\%) had genetic disorders (Table 1).

Lesion types revealed through MRI of the brain included periventricular leukomalacia in 11 patients, disorders of neuronal migration (cortical dysplasia, lissencephaly, polymicrogyria, pachygyria, heterotopia) in seven, cerebral vascular disease in five (including one patient with encephalomalacia suspected due to cerebral vascular disease), brain atrophy in two, and others (venous

Table 1. Patients' demographic characteristics $(n=115)$

\begin{tabular}{|c|c|c|c|}
\hline Characteristic & \multicolumn{3}{|c|}{ Value } \\
\hline \multicolumn{4}{|l|}{ Sex } \\
\hline Male & \multicolumn{3}{|c|}{$68(59)$} \\
\hline Female & \multicolumn{3}{|c|}{$47(41)$} \\
\hline \multicolumn{4}{|l|}{ Age (yr) } \\
\hline Age at seizure onset & \multicolumn{3}{|c|}{$4.88 \pm 2.99$ (4.5 [2.29-6.88]) } \\
\hline Age at first MISF on EEG & \multicolumn{3}{|c|}{$8.08 \pm 3.53(6[5-8.67])$} \\
\hline \multicolumn{4}{|l|}{ Seizure type } \\
\hline Generalized & \multicolumn{3}{|c|}{$49(43)$} \\
\hline Focal & \multicolumn{3}{|c|}{$66(57)$} \\
\hline Perinatal problems & \multicolumn{3}{|c|}{$8(7)$} \\
\hline Status epilepticus & \multicolumn{3}{|c|}{$7(6)$} \\
\hline Lesion on MRI & \multicolumn{3}{|c|}{$29(25)$} \\
\hline Intellectual disability & \multicolumn{3}{|c|}{$68(59)$} \\
\hline Cerebral palsy & \multicolumn{3}{|c|}{$34(30)$} \\
\hline Genetic abnormality & \multicolumn{3}{|c|}{$7(6)$} \\
\hline Follow-up duration (yr) & \multicolumn{3}{|c|}{$5.8 \pm 2.37(6.22$ [3.67-8.03]) } \\
\hline \multicolumn{4}{|c|}{$\begin{array}{l}\text { Values are presented as number }(\%) \text { or mean } \pm \text { standard deviation (media } \\
\text { [interquartile range]). } \\
\text { MISF, multiple independent spike foci; EEG, electroencephalography; MR } \\
\text { magnetic resonance imaging. }\end{array}$} \\
\hline \multicolumn{4}{|c|}{$\begin{array}{l}\text { Table } 2 \text {. Types of lesions on magnetic resonance imaging of th } \\
\text { brain }\end{array}$} \\
\hline Variable & $\begin{array}{l}\text { Group A } \\
(n=18)\end{array}$ & $\begin{array}{c}\text { Group B } \\
(n=11)\end{array}$ & $\begin{array}{c}\text { Total } \\
(n=29)\end{array}$ \\
\hline Encephalomalacia & 6 & 5 & 11 \\
\hline Disorders of neuronal migration & 5 & 2 & 7 \\
\hline Cerebral vascular disease & 3 & 2 & 5 \\
\hline Brain atrophy & 1 & 1 & 2 \\
\hline Venous malformation & 0 & 1 & 1 \\
\hline Tubers & 1 & 0 & 1 \\
\hline $\begin{array}{l}\text { Dysembryoplastic neuroepithelial } \\
\text { tumor }\end{array}$ & 1 & 0 & 1 \\
\hline $\begin{array}{l}\text { Megalencephalic } \\
\text { leukoencephalopathy with } \\
\text { subcortical cyst }\end{array}$ & 1 & 0 & 1 \\
\hline
\end{tabular}

malformation, tubers, dysembryoblastic neuroepithelial tumor, and megalencephalic leukoencephalopathy with subcortical cyst) (Table 2).

The genetic disorders included tuberous sclerosis complex, Prader-Willi syndrome, deletions (deletion of 15q11.2-13.1, 45; XY, del(13, 14)(q10:q10)), 9p trisomy), and Van der Knaap syndrome (megalencephalic leukoencephalopathy with subcortical cyst).

\section{Analysis of risk factors that influence therapeutic result} Among the total 115 patients, 84 (73\%) were in group A and 31 (27\%) were in group B. There was no intergroup difference in sex distribution; both groups showed a male predominance. The mean age at seizure onset was $5.20 \pm 2.92$ years (median age, 4.88 [IQR, 2.83 to 7.19]) in group A versus $4.01 \pm 3.04$ years (median age, 2.50 [IQR, 1.79 to 5.79$]$ ) in group $B$, showing no statistical significance $(\mathrm{P}=0.057)$. The mean age at which MISF was first detected on EEG was 8.15 \pm 3.22 years (median age, 7.96 [IQR, 6 to 9.67]) in group A versus $7.90 \pm 4.33$ years (median age, 7 [IQR, 4.88 to 9.5$]$ ) in group $B$, showing no statistical significance $(\mathrm{P}=0.773)$. The clinical factors of family history of epilepsy and febrile convulsion, history of perinatal problems, and gestational age did not differ between groups (Table 3).

A higher proportion of patients in group $B$ had generalized seizures $(\mathrm{n}=19[61 \%] ; P=0.025)$. The baseline frequency of seizures at the time of MISF detection on EEG was $4.02 \pm 17.52$ times/month (median, 0 times/month [IQR, 0 to 0.33$]$ ) in group $A$ versus $112.23 \pm 357.78$ times/month (median, $1[\mathrm{IQR}, 0$ to $45])$ in group $B(P=0.103)$. The duration from the first seizure to the start of AED did not differ between the groups $(P=0.656)$ (Table 4).

Intellectual disability and cerebral palsy, which were suggested related to symptomatic or cryptogenic epilepsy, were more common in group B $(P<0.001$ and $P=0.046$, respectively). The presence or absence of lesions on MRI did not differ between groups $(P=0.194)$. Six patients in group $A$ versus one patient in group $B$ had a genetic disorder (no statistically significant difference) (Table 5).

In EEG findings, electrodecrements and abnormal background activities such as diffuse slow waves and poorly organized sleep features were more common in group $\mathrm{B}(P=0.005$ and $P=0.003$, respectively) (Table 6).

\section{Clinical course and change in EEG findings with AED therapy}

Total follow-up duration of overall patients was $5.8 \pm 2.37$ years (median, 6.22 [IQR, 3.67 to 8.03]) (Table 7). It was 6.29 \pm 2.22 years (median, 7.21 [IQR, 4.89 to 8.17$]$ ) in group A versus 
Table 3. Patients' clinical profiles according to seizure outcomes

\begin{tabular}{|c|c|c|c|}
\hline Variable & Group A $(n=84)$ & Group B $(n=31)$ & $P$ value \\
\hline \multicolumn{4}{|l|}{ Sex } \\
\hline Male & $49(58.33)$ & $19(61.29)$ & 0.942 \\
\hline Female & 35 (41.67) & $12(38.71)$ & \\
\hline \multicolumn{4}{|l|}{ Age (yr) } \\
\hline Age at seizure onset & $5.20 \pm 2.92(4.8[2.83-7.19])$ & $4.01 \pm 3.04(2.5[1.79-5.79])$ & 0.057 \\
\hline Age at first MISF on EEG & $8.15 \pm 3.22$ (7.96 [6-9.67]) & $7.90 \pm 4.33(7[4.88-9.5])$ & 0.740 \\
\hline Family history of epilepsy & $4(4.76)$ & $2(6.45)$ & 1.000 \\
\hline Family history of febrile convulsion & $2(2.38)$ & $1(3.23)$ & 1.000 \\
\hline Perinatal problem & $6(7.14)$ & $2(6.45)$ & 1.000 \\
\hline Gestational age (yr) & $38.79 \pm 2.86$ & $37.82 \pm 3.64$ & 0.139 \\
\hline
\end{tabular}

Values are presented as number (\%), mean \pm standard deviation (median [interquartile range]), or mean \pm standard deviation.

MISF, multiple independent spike foci; EEG, electroencephalography.

Table 4. Prognostic factors of seizure outcomes associated with seizure profiles

\begin{tabular}{|c|c|c|c|}
\hline Variable & Group A $(n=84)$ & Group B $(n=31)$ & $P$ value \\
\hline Dominant seizure type & & & 0.025 \\
\hline Generalized & $30(35.71)$ & 19 (61.29) & \\
\hline Focal & $50(59.52)$ & $10(32.26)$ & \\
\hline Mixed & $4(4.76)$ & $2(6.45)$ & \\
\hline $\begin{array}{l}\text { Seizure frequency at the time of MISF detection } \\
\text { (times/mo) }\end{array}$ & $4.02 \pm 17.52(0[0-0.33])$ & $112.23 \pm 357.78(1[0-45])$ & 0.103 \\
\hline Status epilepticus & $4(4.76)$ & $3(9.68)$ & 0.590 \\
\hline $\begin{array}{l}\text { Duration between seizure onset and start of } \\
\text { AEDs }\end{array}$ & $0.96 \pm 1.55$ & $0.82 \pm 1.51$ & 0.656 \\
\hline
\end{tabular}

Values are presented as number (\%), mean \pm standard deviation (median [interquartile range]), or mean \pm standard deviation.

MISF, multiple independent spike foci; AED, anti-epileptic drug.

Table 5. Lesion findings on MRI and comorbidities as prognostic factors of seizure outcomes

\begin{tabular}{|c|c|c|c|}
\hline Variable & Group A $(n=84)$ & Group B $(n=31)$ & $P$ value \\
\hline \multicolumn{4}{|l|}{ Etiology $(n=115)$} \\
\hline Presence of lesion on MRI & $18(21.43)$ & $12(38.7)$ & 0.194 \\
\hline \multicolumn{4}{|l|}{ Comorbidity $(n=115)$} \\
\hline Intellectual disability & $40(47.62)$ & $28(90.32)$ & $<0.001$ \\
\hline Cerebral palsy & $20(23.81)$ & $14(45.16)$ & 0.046 \\
\hline Genetic abnormality & $6(7.14)$ & $1(3.23)$ & 0.734 \\
\hline
\end{tabular}

Values are presented as number (\%).

$\mathrm{MRI}$, magnetic resonance imaging.

Table 6. Correlations between electroencephalography findings other than multiple independent spike foci and seizure outcomes

\begin{tabular}{lccc}
\hline Variable & Group A $(n=84)$ & Group B $(n=31)$ & $4(12.90)$ \\
\hline Generalized epileptiform discharge & $8(9.52)$ & $7(22.58)$ & 0.855 \\
Electrodecrements & $3(3.57)$ & $22(70.97)$ & 0.005 \\
Abnormal background activity & $32(38.10)$ & $18(58.06)$ & 0.003 \\
$\quad$ Diffuse slow waves & $17(20.24)$ & $3(9.68)$ & $<0.001$ \\
Focal slow waves or asymmetry & $3(3.57)$ & 0.404 \\
\hline
\end{tabular}

Values are presented as number (\%). 
$4.48 \pm 2.30$ years (median, 4.07 [IQR, 2.62 to 6.14]) (Table 7). In group A, they had seizure-free period of $3.87 \pm 2.47$ years (median, 3.14 [IQR, 2.06 to 5.71$]$ ) by last follow-up visit.

A higher number of patients received polytherapy of AED in group A $(n=32[38.10 \%])$ than in group B $(n=24[77.42 \%])$ $(P=0.001)$. The mean number of AEDs used was $1.52 \pm 0.91$ in group A versus $3.42 \pm 1.98$ in group $B(P<0.001)$ (Table 7$)$.

The number of patients treated with anti-seizure therapy other than AED therapy such as a ketogenic diet or vagus nerve stimulation (VNS) was higher in group $\mathrm{B}$ than group $\mathrm{A}(P<0.001$ and $P=0.018$ ) (Table 7).

The findings of follow-up EEG showed improvements in MISF along with improvements in clinical seizure. Among total $109 \mathrm{pa}-$ tients who had follow-up EEG, 81 were in group $A$ and 28 were in group B. More patients in group A $(n=58[71.6 \%])$ than group B $(n=10[35.7 \%])$ showed the disappearance of MISF on EEG. Furthermore, EEG findings were normal in more patients in group $A(n=21[25.9 \%])$ than in group $B(n=0[0 \%])$ $(P=0.007)$ (Table 8$)$.

\section{Discussion}

Very few studies have described the characteristics of patients with MISF since the study of Blume et al. [1,4] in 1970s. The current study aimed to elucidate the clinical characteristics and prognostic factors of patients with post-infantile epilepsy with good prognosis despite the presence of MISF on EEG. For this purpose, this study excluded patients with a history of infantile spasms, a relatively wellknown epileptic syndrome with remarkable characteristics and poor prognosis. As a result, we included a considerable number of patients with MISF on EEG who had post-infantile epilepsy and a good response to AED therapy showing improvement in clinical seizures as high as two-thirds (group A, $\mathrm{n}=84$ [73\%]). Patients in the seizure control group required fewer AEDs $(1.52 \pm 0.91)$ and were less likely to require adjuvant therapies such as a ketogenic diet and VNS.

In our study, the prognostic factors associated with a poor prognosis and poor seizure control were generalized seizures, premorbid intellectual disability or cerebral palsy, and EEG abnormalities such as electrodecrements and abnormal background activity.

Blume et al. [4] reported that half of their studied patients with MISF had daily and frequent seizures, while one-third had moderate to severe intellectual disability and were difficult to educate. They concluded that these were descriptive characteristics of patients with MISF, while the current study revealed that frequent seizures and intellectual disability were features highly suggestive of a poor prognosis but not characteristics of MISF specifically.

In the literature, the usefulness of EEG is well documented. For example, the EEG abnormality supports the prediction of seizure recurrence in the first unprovoked seizure and the diagnosis of epilepsy [7-9]. In addition to it, our study suggests two aspect of usefulness of EEG. First, abnormal findings additional to MISF are correlated with prognosis. Background abnormalities such as diffuse slow waves or abnormal sleep features were highly suggestive

Table 7. Differences in the clinical course of post-infantile patients with epilepsy and MISF on EEG

\begin{tabular}{|c|c|c|c|}
\hline Variable & Group A $(n=84)$ & Group B $(n=31)$ & $P$ value \\
\hline Follow-up duration (yr) & $6.29 \pm 2.22(7.21[4.89-8.17])$ & $4.48 \pm 2.30(4.07[2.62-6.14])$ & \\
\hline Seizure-free duration (yr) & $3.87 \pm 2.47(3.14[2.06-5.71])$ & - & \\
\hline Number of AEDs used in treatment & $1.52 \pm 0.91$ & $3.42 \pm 1.98$ & $<0.001$ \\
\hline Monotherapy vs. polytherapy & & & 0.001 \\
\hline Monotherapy & $52(61.90)$ & $7(22.58)$ & \\
\hline Polytherapy & $32(38.10)$ & $24(77.42)$ & \\
\hline VNS & 0 & 3 (9.68) & 0.018 \\
\hline Ketogenic diet & $1(1.19)$ & $9(29.03)$ & $<0.001$ \\
\hline
\end{tabular}

Values are presented as mean \pm standard deviation (median [interquartile range]), mean \pm standard deviation, or number (\%). MISF, multiple independent spike foci; EEG, electroencephalography; AED, anti-epileptic drug; VNS, vagus nerve stimulation.

Table 8. Differences in follow-up EEG findings of post-infantile patients with epilepsy and MISF on EEG

\begin{tabular}{lccc}
\hline Follow-up EEG findings $(n=109)$ & Group $A(n=84)$ & Group B $(n=31)$ & $P$ value \\
\hline Disappearance of MISF & $58(71.6)$ & $10(35.7)$ & 0.002 \\
Normalization & $21(25.9)$ & 0 & 0.007 \\
\hline
\end{tabular}

Values are presented as number (\%).

EEG, electroencephalography; MISF, multiple independent spike foci. 
of poor prognosis in seizure control. Second, the improvement on EEG reflected the clinical progression of improvement. Our study showed that normalization or improvement of MISF on follow-up EEG was highly correlated with an improving clinical course. Twothirds $(72.6 \%)$ of the seizure control group was accompanied by improvement in MISF on EEG and one-fourth (25.9\%) of the seizure control group had fully normalized EEG findings with AED therapy. We suggest that EEG is useful for predicting prognosis of seizure control and estimating clinical improvement with AED therapy.

A recent analysis of MRI lesions and EEG findings in epilepsy patients described that patients with multifocal interictal epileptiform discharges on EEG had a statistically significantly higher rate (68\%) of abnormalities on MRI [10]. This was somewhat different from our result that $25 \%$ of patients have a lesion on MRI, similar to the $21 \%$ to $31 \%$ of the overall pediatric patients who visited with a first unprovoked seizure [11-14]. In cases of pediatric epilepsy, the rate of abnormalities on brain MRI increased [10]. The reasons that our study findings suggested that the presence of a lesion on MRI was not significantly associated with seizure control might be due to patients with significantly severe lesions being excluded by exclusion criteria of whom diagnosed infantile spasms and early-onset seizures before 1 year of age; thus, a relatively small number of patients with less severe abnormalities on brain MRI were included in our study.

Blume et al. [1] reported that a considerable number of patients with findings of MISF on EEG had generalized seizures. Several strudies described that the major seizure type of SE-MISF was brief generalized tonic seizure [5,15-17]. Conversely, in the current study, the percentage of patients with focal seizures was higher than previously reported, while the focal seizure type was a proven good prognostic factor for seizure control. This suggests that seizure type is a major clinical characteristic that influences prognosis.

Since 1992, several studies suggested that epileptic patients with clinical characteristics, especially a severe clinical course and MISF on EEG could be defined as an epileptic syndrome [5,15-18], primarily LGS or SE-MISF, which differs in several ways. In 2006, Yamatogi and Ohtahara [6] defined SE-MISF and summarized its characteristics [6]. SE-MISF is defined as symptomatic generalized epilepsy with age-specific epileptic encephalopathy. They described the characteristics of SE-MISF as: (1) MISF, diffuse slow background activity, and rare diffuse epileptic discharges on EEG; (2) frequent generalized minor seizures as the main seizure type; (3) mutual transition between the age-dependent epileptic encephalopathies (i.e., Ohtahara syndrome, West syndrome, and LGS); (4) early onset of epilepsy (despite variable SE-MISF onset); (5) association with intellectual disability and neurological abnormality; (6) variable and non-specific etiology; and (7) intractable seizure and psychomotor deterioration.

Likewise, the current study showed that the characteristics associated with a poor prognosis were abnormal background activity on EEG, generalized seizures, and intellectual disability. These are similar to the characteristics of SE-MISF and accounted for three of seven characteristics mentioned by Yamatogi and Ohtahara [6].

Despite SE-MISF not being widely accepted as an epileptic syndrome, efforts to categorize patients with MISF as epileptic syndromes are increasing with our understanding of them. Similarly, our study emphasized that MISF on EEG is not strongly correlated with LGS, SE-MISF, or intractable seizures. Thus, we conclude that MISF should be interpreted correctly and precisely according to individual clinical characteristics, which would prevent errors in the estimation of prognosis during diagnosis and the early phase of treatment.

In current study, seizure control was defined as seizure freedom over 6 months by the last visit. This definition might reflect a shortterm outcome in some patients, but in fact the subject had various follow-up periods and seizure-free periods because this study was retrospective. If a prospective study with a long-term follow-up period is conducted, we can further understand the characteristics of benign and severe epileptic patients with MISF and more concise statistics related to the prognosis, such as seizure recurrence, progression of seizure frequency of epileptic encephalopathy, possibility of AED cessation, a long-term seizure-free period, and finally epilepsy cure.

A further study is required to elucidate the clinical significance of MISF in patients with good prognosis, particularly the pathogenesis of epilepsy in cases of idiopathic generalized epilepsy or focal onset seizures.

\section{Conflicts of interest}

No potential conflict of interest relevant to this article was reported.

\section{ORCID}

Juhyun Kong, http://orcid.org/0000-0001-5900-5103

Sang Ook Nam, http://orcid.org/0000-0003-1312-3483

\section{Author contribution}

Conceptualization: JK and SON. Data curation: JK, YJL, AK, YMK, and GMY. Formal analysis: JK and YJL. Methodology: JK and SON. Project administration: JK, YJL, AK, GMY, and SON. Visualization: JK. Writing-original draft: JK. Writing-review \& editing: JK and SON. 


\section{Acknowledgments}

This work was supported by a 2-Year Research Grant of Pusan National University.

\section{References}

1. Blume WT, David RB, Gomez MR. Generalized sharp and slow wave complexes. Associated clinical features and long-term follow-up. Brain 1973;96:289-306.

2. Friedman E, Pampiglione G. Prognostic implications of electroencephalographic findings of hypsarrhythmia in first year of life. Br Med J 1971;4:323-5.

3. Noriega-Sanchez A, Markand ON. Clinical and electroencephalographic correlation of independent multifocal spike discharges. Neurology 1976;26:667-72.

4. Blume WT. Clinical and electroencephalographic correlates of the multiple independent spike foci pattern in children. Ann Neurol 1978;4:541-7.

5. Yamatogi Y, Ohtahara S. Severe epilepsy with multiple independent spike foci. J Clin Neurophysiol 2003;20:442-8.

6. Yamatogi Y, Ohtahara S. Multiple independent spike foci and epilepsy, with special reference to a new epileptic syndrome of "severe epilepsy with multiple independent spike foci”. Epilepsy Res 2006;70 Suppl 1:S96-104.

7. Berg AT, Shinnar S. The risk of seizure recurrence following a first unprovoked seizure: a quantitative review. Neurology 1991;41:965-72.

8. Pohlmann-Eden B, Newton M. First seizure: EEG and neuroimaging following an epileptic seizure. Epilepsia 2008;49 Suppl $1: 19-25$.

9. Bouma HK, Labos C, Gore GC, Wolfson C, Keezer MR. The diagnostic accuracy of routine electroencephalography after a first unprovoked seizure. Eur J Neurol 2016;23:455-63.

10. Dirik MA, Sanlidag B. Magnetic resonance imaging and interictal electroencephalography findings in newly diagnosed epileptic children. J Clin Med 2018;7:134.

11. Gaillard WD, Chiron C, Cross JH, Harvey AS, Kuzniecky R, Hertz-Pannier L, et al. Guidelines for imaging infants and children with recent-onset epilepsy. Epilepsia 2009;50:2147-53.

12. Tews W, Weise S, Syrbe S, Hirsch W, Viehweger A, Merkenschlager A, et al. Is there a predictive value of EEG and MRI after a first afebrile seizure in children? Klin Padiatr 2015;227:848.

13. Shinnar S, O’Dell C, Mitnick R, Berg AT, Moshe SL. Neuroimaging abnormalities in children with an apparent first unprovoked seizure. Epilepsy Res 2001;43:261-9.

14. Sharma S, Riviello JJ, Harper MB, Baskin MN. The role of emergent neuroimaging in children with new-onset afebrile seizures. Pediatrics 2003;111:1-5.

15. Ohtsuka Y, Amano R, Mizukawa M, Ohtahara S. Long-term prognosis of the Lennox-Gastaut syndrome. Jpn J Psychiatry Neurol 1990;44:257-64.

16. Ohtsuka Y, Amano R, Mizukawa M, Maniwa S, Ohtahara S. Long-term prognosis of the Lennox-Gastaut syndrome: consideration in its evolutional change. In: Fukuyama Y, Kamoshita S, Ohtsuka C, Suzuki Y, editors. Modern perspectives of child neurology. Tokyo: The Japanese Society of Child Neurology; 1991.p. 215-22.

17. Ohtahara S, Ohtsuka Y, Kobayashi K. Lennox-Gastaut syndrome: a new vista. Psychiatry Clin Neurosci 1995;49:S179-83.

18. Mizukawa M. Severe epilepsy with multiple independent spike foci: a clinical and electroencephalographic study. J Jpn Epil Soc 1992;10:78-87. 